\title{
OPTIMAL FUZZY CONTROL USING HEDGE ALGEBRAS OF A DAMPED ELASTIC JOINTED INVERTED PENDULUM
}

\author{
Bui Hai Le ${ }^{1}$, Tran Duc Trung ${ }^{1}$ and Vu Nhu Lan ${ }^{2}$ \\ ${ }^{1}$ Hanoi University of Science and Technology \\ ${ }^{2}$ Institute of Information Technology
}

\begin{abstract}
In this paper, three controllers including OFCHA (optimal fuzzy control using hedge algebras - HAs), FCHA (fuzzy control using HAs) and CFC (conventional fuzzy control) are designed. Our attention is paid to the stability in the vertical position of a damped-elastic-jointed inverted pendulum subjected to a time periodic follower force. Different values of the pendulum length are considered. Simulation results are exposed to illustrate the effect of OFCHA in comparison with FCHA and CFC.
\end{abstract}

\section{INTRODUCTION}

Fuzzy set theory introduced by Zadeh in 1965 has provided a mathematical tool useful for modelling uncertain (imprecise) and vague data and been presented in many real situations. As typical unstable nonlinear models, inverted pendulum systems are often used as a benchmark for verifying the effect of a new control method because of the simplicity of the structure. Recently, many researches on stabilization of pendulum systems have been done.

Yi and Yubazaki [1] presented a fuzzy controller for stabilization control of inverted pendulum systems basing on the Single Input Rule Modules (SIRMs) dynamically connected fuzzy inference model. The fuzzy controller has four input items, each with a SIRM and a dynamic importance degree. Yi et al. [2] presented a fuzzy controller with six input items and one output item for stabilizing a parallel-type double inverted pendulum system basing on the SIRMs dynamically connected fuzzy inference model. Becerikli and Celikb [3] presented a fuzzy controller for an inverted pendulum system in two stages: investigation of fuzzy control system modeling methods and solution of the "Inverted Pendulum Problem" by using Java programming with Applets for internet based control education. Tao et al. [4] proposed a fuzzy hierarchical swing-up and sliding position controller for the swing-up and position controls of an inverted pendulum-cart system which includes a fuzzy switching controller, a fuzzy swing-up controller, and a twin-fuzzy-sliding-position controller. $\mathrm{Li}$ and $\mathrm{Xu}[5]$ investigated adaptive fuzzy logic control of dynamic balance and motion is for wheeled inverted pendulums with parametric and functional uncertainties. The proposed adaptive fuzzy logic control based on physical properties of wheeled inverted 
pendulums makes use of a fuzzy logic engine and a systematic online adaptation mechanism to approximate the unknown dynamics. Sinha and Butcher [6] used a technique that employs both Picard iteration and expansion in shifted Chebyshev polynomials to symbolically approximate the fundamental solution matrix for linear time-periodic dynamical systems of arbitrary dimension explicitly as a function of the system parameters and time. A double inverted pendulum subjected to a periodic follower force was considered as an example. Galán et al. [7] studied a chain of identical pendulums coupled damped elastic joints subject to vertical sinusoidal forcing of its base. Particular attention was paid to the stability of the upright equilibrium configuration with a view to understanding recent experimental results on the stabilization of an unstable stiff column under parametric excitation. Ma and Butcher [8] presented a stability analysis for elastic columns under the influence of periodically varying follower forces whose orientation is retarded, i.e., depends on the position of the system at a previous time. Mailybaev and Seyranian [9] studied the stability of a linear vibrational system with multi-degrees-of-freedom subjected to parametric excitation. A double inverted pendulum subjected to periodic excitation of support was considered as an example.

In this paper, three controllers including OFCHA (optimal fuzzy control using hedge algebras - HAs), FCHA (fuzzy control using HAs) and CFC (conventional fuzzy control) are designed.

Although a CFC is flexible and easy in use, but its semantic order of linguistic values is not closely guaranteed and its fuzzification and defuzzification methods are quite complicated.

HAs was introduced and investigated since 1987 ([10-15]). The authors of HAs discovered that: linguistic values can formulate an algebraic structure $[10,11]$ and it is a Complete Hedge Algebras Structure $[13,14]$ with a main property is that the semantic order of linguistic values is always guaranteed. It is even a rich enough algebraic structure [12] and, therefore, it can describe completely reasoning processes. In [15], HAs theory was begun applying to fuzzy control and it provided very much better results than CFC, objects studied in [15], however, were too simple to evaluate completely its control effect.

That reason suggests us, in this paper, applying HAs in fuzzy control for stabilizing a damped-elastic-jointed inverted pendulum subjected to a time periodic follower force with three controllers (CFC, FCHA and OFCHA) in order to compare their control effect.

\section{THE DAMPED-ELASTIC-JOINTED INVERTED PENDULUM}

The diagram of the system is shown in Fig. 1. An inverted pendulum of length $l$ has a concentrated mass $m$ at its end. A spring (of constant stiffness $k$ ) and a damper (of constant damping factor $c$ ) connect the inverted pendulum to a fixed base to avoid large rotation angle $\varphi$. A periodic follower force $\left(F=P_{1}+P_{2} \cos \omega t\right)$ acts on the mass $m$ and makes an angle $\gamma \varphi$ with the vertical axis. A control torque $u$, applied at the base of the pendulum, brings the angle $\varphi$ to zero position.

The kinetic energy $T$, the potential energy $\Pi$ and the dissipative function $\Phi$ of the system can be expressed in the following forms (Galán et al. [7]):

$$
\mathrm{T}=\frac{1}{2} m\left(\dot{x}_{m}^{2}+\dot{y}_{m}^{2}\right)=\frac{1}{2} m \dot{\varphi}^{2} l^{2}
$$




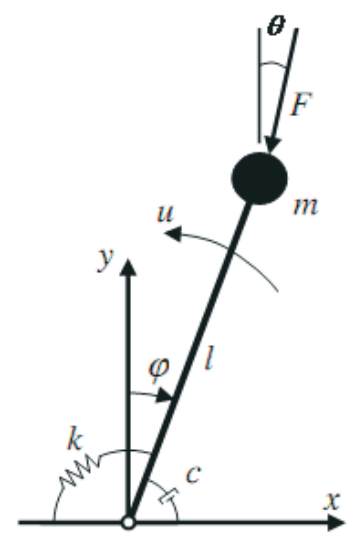

Fig. 1. Configuration of the inverted pendulum system

$$
\begin{gathered}
\Pi=\frac{1}{2} m g y_{m}+\frac{1}{2} k \varphi^{2}=\frac{1}{2} m g l \cos \varphi+\frac{1}{2} k \varphi^{2} \\
\Phi=\frac{1}{2} c \dot{\varphi}^{2}
\end{gathered}
$$

where, $x_{m}=l \sin \varphi ; y_{m}=l \cos \varphi$ and $g$ is the gravity acceleration.

Using the Lagrange equations of the second type:

$$
\frac{d}{d t}\left(\frac{\partial \mathrm{T}}{\partial \dot{\varphi}}\right)-\frac{\partial \mathrm{T}}{\partial \varphi}=-\frac{\partial \Pi}{\partial \varphi}-\frac{\partial \Phi}{\partial \varphi}+\mathrm{M}
$$

The differential equation of motion for this system can then be written in the form:

$$
m l^{2} \ddot{\varphi}+c \dot{\varphi}+k \varphi-\frac{1}{2} m g l \sin \varphi=u+F l \sin (\varphi-\theta)
$$

where the angle $\theta$ is shown in the Fig. 1.

Denoting $\left[x_{1}, x_{2}\right]^{\mathrm{T}}=[\varphi, \dot{\varphi}]^{\mathrm{T}}$, Eq. (5) can be rewritten into state-space form as follow:

$$
\begin{aligned}
& \dot{x}_{1}=\dot{\varphi}=x_{2} \\
& \dot{x}_{2}=\ddot{\varphi}=-\frac{k}{m l^{2}} x_{1}+\frac{g}{2 l} \sin x_{1}+\frac{F l \sin \left(x_{1}-\gamma x_{1}\right)}{m l^{2}}-\frac{c}{m l^{2}} x_{2}+\frac{u}{m l^{2}}
\end{aligned}
$$

The Eqs. (6) can be solved by using the fourth-order Runge-Kutta method (Chapra and Canale [16]).

\section{HEDGE ALGEBRAS}

In this section, the idea and basic formulas of HAs are summarized based on definitions, theorems, propositions in [10-15].

By the term meaning we can observe that extremely small $<$ very small $<$ small $<$ approximately small $<$ little small $<$ big $<$ very big $<$ extremely big... So, we have a new viewpoint: term-domains can be modelled by a poset (partially ordered set), a semanticsbased order structure. Next, we explain how we can find out this structure. 
Consider TRUTH as a linguistic variable and let $X$ be its term-set. Assume that its linguistic hedges used to express the TRUTH are Extremely, Very, Approximately, Little, which for short are denoted correspondingly by $E, V, A$ and $L$, and its primary terms are false and true. Then, $X=\{$ true, $V$ true, E true, EA true, $A$ true, LA true, $L$ true, $L$ false, false, A false, $V$ false, $E$ false $\ldots \cup\{\boldsymbol{O}, \boldsymbol{W}, \boldsymbol{1}$ is a term-domain of TRUTH, where $\boldsymbol{O}, \boldsymbol{W}$ and $\boldsymbol{1}$ are specific constants called absolutely false, neutral and absolutely true, respectively.

A term-domain $X$ can be ordered based on the following observation:

- Each primary term has a sign which expresses a semantic tendency. For instance, true has a tendency of "going up", called positive one, and it is denoted by $c^{+}$, while false has a tendency of "going down", called negative one, denoted by $c^{-}$. In general, we always have $c^{+} \geq c^{-}$, semantically.

- Each hedge has also a sign. It is positive if it increases the semantic tendency of the primary terms and negative, if it decreases this tendency. For instance, $V$ is positive with respect to both primary terms, while $L$ has a reverse effect and hence it is negative. Denote by $H^{-}$the set of all negative hedges and by $H^{+}$the set of all positive ones of TRUTH.

The term-set $X$ can be considered as an abstract algebra $A X=(X, G, C, H, \leq)$, where $G=\left\{c^{-}, c^{+}\right\}, C=\{\boldsymbol{o}, \boldsymbol{W}, \boldsymbol{1}\}, H=H^{+} \cup H^{-}$and $\leq$is a partially ordering relation on $X$. It is assumed that $H^{-}=\left\{h_{-1}, \ldots, h_{-q}\right\}$, where $h_{-1}<h_{-2}<\ldots<h_{-q}$, $H^{+}=\left\{h_{1}, \ldots, h_{p}\right\}$, where $h_{1}<h_{2}<\ldots<h_{p}$.

Fuzziness measure of vague terms and hedges of term-domains is defined as follow (Definition 2 - [15]): a $\mathrm{fm}: X \rightarrow[0,1]$ is said to be a fuzziness measure of terms in $X$ if:

$$
f m\left(c^{-}\right)+f m\left(c^{+}\right)=1 \text { and } \sum_{h \in H} f m(h u)=f m(u), \text { for } \forall u \in X,
$$

For the constants $\boldsymbol{O}, \boldsymbol{W}$ and $\mathbf{1}$,

$$
f m(\boldsymbol{O})=f m(\boldsymbol{W})=f m(\boldsymbol{1})=0,
$$

For $\forall x, y \in X, \forall h \in H$,

$$
\frac{f m(h x)}{f m(x)}=\frac{f m(h y)}{f m(y)}
$$

This proportion does not depend on specific elements, called fuzziness measure of the hedge $h$ and denoted by $\mu(h)$.

For each fuzziness measure $f m$ on $X$, we have (Proposition 1 - [15]):

$$
\begin{gathered}
f m(h x)=\mu(h) f m(x), \text { for every } x \in X, \\
f m\left(c^{-}\right)+f m\left(c^{+}\right)=1, \\
\sum_{i=-q, i \neq 0}^{p} f m\left(h_{i} c\right)=f m(c), c \in\left\{c^{-}, c^{+}\right\}, \\
\sum_{i=-q, i \neq 0}^{p} f m\left(h_{i} x\right)=f m(x) ;
\end{gathered}
$$




$$
\sum_{i=-q}^{-1} \mu\left(h_{i}\right)=\alpha \text { and } \sum_{i=1}^{p} \mu\left(h_{i}\right)=\beta \text { where } \alpha, \beta>0 \text { and } \alpha+\beta=1
$$

A function Sign: $X \rightarrow\{-1,0,1\}$ is a mapping which is defined recursively as follows, for $h, h^{\prime} \in H$ and $c \in\left\{c^{-}, c^{+}\right\}$(Definition $3-[15]$ ):

$$
\operatorname{Sign}\left(c^{-}\right)=-1, \operatorname{Sign}\left(c^{+}\right)=+1,
$$

$\operatorname{Sign}(h c)=-\operatorname{Sign}(c)$, if $h$ is negative w.r.t. $c$,

$$
\operatorname{Sign}(h c)=+\operatorname{Sign}(c) \text {, if } h \text { is positive w.r.t. } c \text {, }
$$

$\operatorname{Sign}\left(h^{\prime} h x\right)=-\operatorname{Sign}(h x)$, if $h^{\prime} h x \neq h x$ and $h^{\prime}$ is negative w.r.t. $h$,

$\operatorname{Sign}\left(h^{\prime} h x\right)=+\operatorname{Sign}(h x)$, if $h^{\prime} h x \neq h x$ and $h^{\prime}$ is positive w.r.t. $h$,

$$
\operatorname{Sign}\left(h^{\prime} h x\right)=0 \text {, if } h^{\prime} h x=h x .
$$

Let $f m$ be a fuzziness measure on $X$. A semantically quantifying mapping (SQM) $\varphi: X \rightarrow[0,1]$, which is induced by $f m$ on $X$, is defined as follows (Definition $4-[15]$ ):

$$
\begin{gathered}
\varphi(\boldsymbol{W})=\theta=f m\left(c^{-}\right), \varphi\left(c^{-}\right)=\theta-\alpha f m\left(c^{-}\right)=\beta f m\left(c^{-}\right), \varphi\left(c^{+}\right)=\theta+\alpha f m\left(c^{+}\right), \\
\varphi\left(h_{j} x\right)=\varphi(x)+\operatorname{Sign}\left(h_{j} x\right)\left\{\sum_{i=\operatorname{Sign}(j)}^{j} f m\left(h_{i} x\right)-\omega\left(h_{j} x\right) f m\left(h_{j} x\right)\right\},
\end{gathered}
$$

where

$$
j \in\{j:-q \leq j \leq p \& j \neq 0\}=\left[-q^{p}\right]
$$

and

$$
\omega\left(h_{j} x\right)=\frac{1}{2}\left[1+\operatorname{Sign}\left(h_{j} x\right) \operatorname{Sign}\left(h_{p} h_{j} x\right)(\beta-\alpha)\right] .
$$

It can be seen that the mapping $\varphi$ is completely defined by $(p+q)$ free parameters: one parameter of the fuzziness measure of a primary term and $(p+q-1)$ parameters of the fuzziness measure of hedges.

To illustrate a close relationship between the meaning of terms and their fuzziness measure and the way to compute semantically quantifying mappings, we consider the following example.

Example: Consider a hedge algebra $A X=(X, G, C, H, \leq)$, where $G=\{$ small, large $\}$, $C=\{\boldsymbol{o}, \boldsymbol{W}, \boldsymbol{1}\}, H^{-}=\{$Little $\}=\left\{h_{-1}\right\}, q=1, H^{+}=\{$Very $\}=\left\{h_{1}\right\}, p=1$. We assume:

$$
\theta=0.5, \quad \alpha=0.5 \text {. }
$$

It means that the semantically quantifying mapping of the neutral element and the sum of the fuzziness measure of the negative hedges are 0.5. Hence,

- Using Equation (14) with $q=1$, we have fuzziness measures of the hedges:

$$
\mu(\text { Little })=\alpha=0.5 ; \quad \mu(\text { Very })=\beta=1-\alpha=0.5 ;
$$

- Next, using Equations (21) and (11), we have fuzziness measures of the terms:

$$
f m(\operatorname{small})=\theta=0.5 ; \quad f m(\text { large })=1-f m(\text { small })=0.5 ;
$$


- Then, semantically quantifying mappings of the linguistic values are computed by using Equations (21) and (22) as follow:

$$
\begin{aligned}
& \varphi(\boldsymbol{W})=\theta=0.5 ; \\
& \varphi(\text { small })=\theta-\alpha f m(\text { small })=0.5-0.5 \times 0.5=0.25 ; \\
& \begin{aligned}
\varphi(\text { Very small }) & =\varphi(\text { small })+\operatorname{Sign}(\text { Very small }) \times(f m(\text { Very small })-0.5 f m(\text { Very small })) \\
& =0.25+(-1) \times 0.5 \times 0.5 \times 0.5=0.125 ;
\end{aligned} \\
& \begin{aligned}
\varphi(\text { Little small }) & =\varphi(\text { small })+\operatorname{Sign}(\text { Little small }) \times(f m(\text { Little small })-0.5 f m(\text { Little small })) \\
& =0.25+(+1) \times 0.5 \times 0.5 \times 0.5=0.375 ;
\end{aligned} \\
& \begin{aligned}
\varphi(\text { large })=\theta+ & \alpha f m(\text { large })=0.5+0.5 \times 0.5=0.75 ; \\
\varphi(\text { Very large }) & =\varphi(\text { large })+\operatorname{Sign}(\text { Very large }) \times(f m(\text { Very large })-0.5 f m(\text { Very large })) \\
& =0.75+(+1) \times 0.5 \times 0.5 \times 0.5=0.875 ;
\end{aligned} \\
& \begin{aligned}
\varphi(\text { Littlelarge }) & =\varphi(\text { large })+\operatorname{Sign}(\text { Littlelarge }) \times(\text { fm }(\text { Littlelarge })-0.5 f m(\text { Little large })) \\
& =0.75+(-1) \times 0.5 \times 0.5 \times 0.5=0.625 .
\end{aligned}
\end{aligned}
$$

The results obtained from above example will be used in the following section.

\section{FUZZY CONTROL SYSTEMS OF THE INVERTED PENDULUM}

The fuzzy control systems are based on the closed-loop fuzzy system shown in Fig. 2. Where, $u$ is determined by above-mentioned controllers, $x_{1}$ and $x_{2}$ are determined by Equations (6). It is assumed that the universe of discourse for the two state variables and control torque to be $-1 \mathrm{rad} \leq x_{1} \leq 1 \mathrm{rad},-2 \mathrm{rad} / \mathrm{s} \leq x_{2} \leq 2 \mathrm{rad} / \mathrm{s}$ and $-50 \mathrm{Nm} \leq u \leq 50$ $\mathrm{Nm}$, respectively. In the following parts of this section, establishing steps of the controllers will be presented.

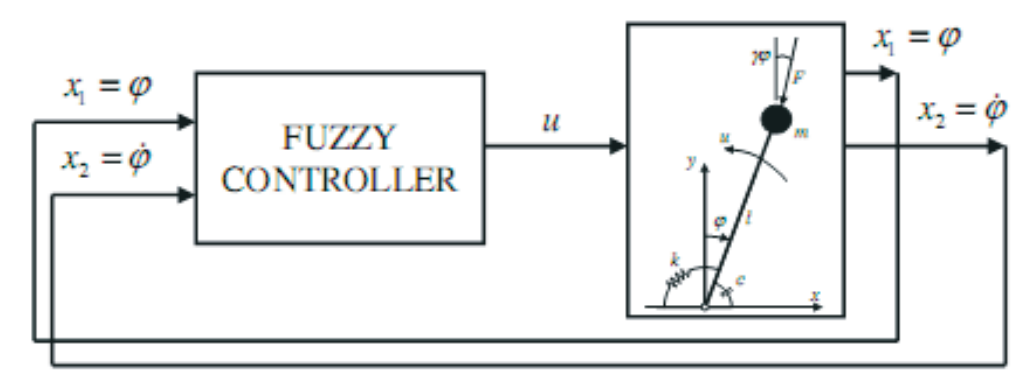

Fig. 2. The inverted pendulum fuzzy control systems

\subsection{Conventional fuzzy control (CFC) of the inverted pendulum}

In this subsection, the CFC of the structure is established (establishing steps of a CFC could refer in Ross [17]) using Mamdani's inference and centroid defuzzification method with nine control rules. The con?guration of the CFC is shown in Fig. 3. 


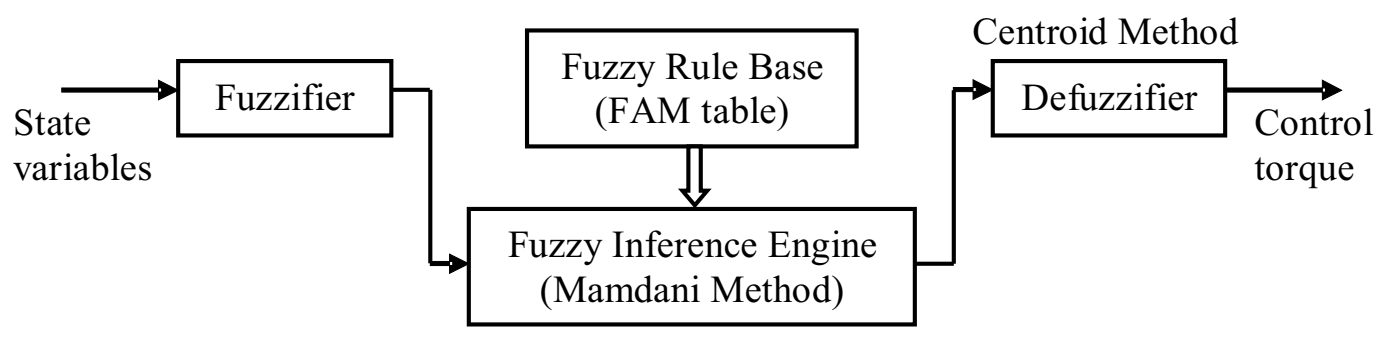

Fig. 3. The configuration of the CFC

\subsubsection{Fuzzifier}

The membership functions of $x_{1}, x_{2}$ and $u$ on their universes, that are, for the values negative big $(\mathrm{NB})$, negative $(\mathrm{N})$, zero $(\mathrm{Z})$, positive $(\mathrm{P})$, positive big $(\mathrm{PB})$ are established as shown in Figs. 4-6, respectively.

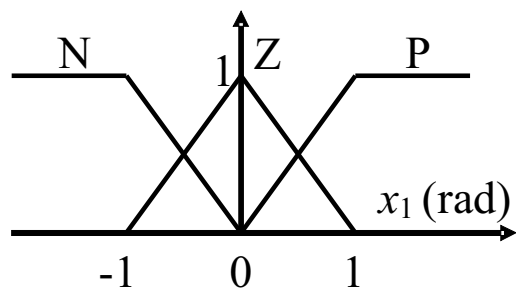

Fig. 4. Input $x_{1}$, partitioned

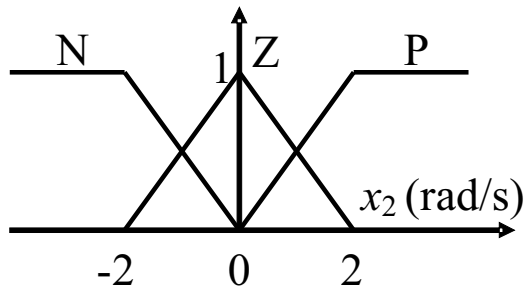

Fig. 5. Input $x_{2}$, partitioned

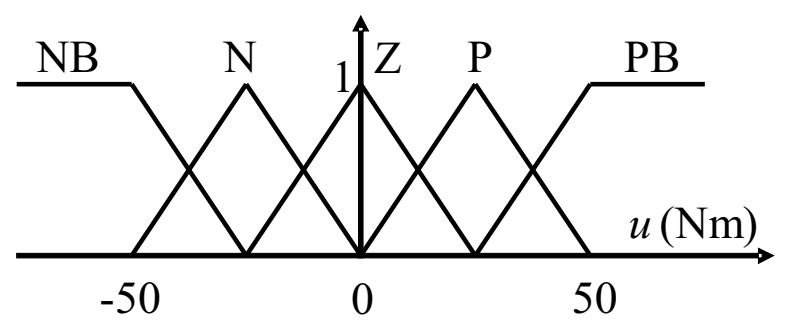

Fig. 6. Output $u$, partitioned

\subsubsection{Fuzzy rule base}

The fuzzy associative memory tables (FAM tables) are established as shown in Table 1.

\subsection{Fuzzy control using hedge algebras (FCHA) of the inverted pendulum}

In CFC, the FAM table is formulated in Table 1. The linguistic labels in Table 1 have to be transformed into the new one given in Table 2, that are suitable to describe linguistically reference domains of $[0,1]$ and can be modeled by suitable HAs. The HAs of the state variables $x_{1}$ and $x_{2}$ are $A X=(X, G, C, H, \leq)$, where $X=x_{1}$ or $x_{2}, G=$ 
Table 1. FAM table for pendulum fuzzy control system rule base

\begin{tabular}{|c|c|c|c|}
\hline \multirow{2}{*}{$x_{1}$} & \multicolumn{3}{|c|}{$x_{2}$} \\
\cline { 2 - 4 } & $\mathrm{N}$ & $\mathrm{Z}$ & $\mathrm{P}$ \\
\hline $\mathrm{N}$ & $\mathrm{NB}$ & $\mathrm{N}$ & $\mathrm{Z}$ \\
\hline $\mathrm{Z}$ & $\mathrm{N}$ & $\mathrm{Z}$ & $\mathrm{P}$ \\
\hline $\mathrm{P}$ & $\mathrm{Z}$ & $\mathrm{P}$ & $\mathrm{PB}$ \\
\hline
\end{tabular}

$\{$ small, large $\}, C=\{\boldsymbol{o}, \boldsymbol{W}, \mathbf{1}\}, H=\left\{H^{-}, H^{+}\right\}=\{$Little, Very $\}$, and the HAs of the control variables $A U=(u, G, C, H, \leq)$ with the same sets $G, C$ and $H$ as for $x_{1}$ and $x_{2}$, however, their terms describe different quantitative semantics based on different real reference domains.

Table 2. Terms transformation

\begin{tabular}{|c|c|c|c|c|}
\hline NB & $\mathrm{N}$ & $\mathrm{Z}$ & $\mathrm{P}$ & $\mathrm{PB}$ \\
\hline Very small & small & $\boldsymbol{W}$ & large & Very large \\
\hline
\end{tabular}

The semantically quantifying mappings (SQMs) $\varphi$ are determined and shown in Table 3 (see section 3 ).

Table 3. Parameters of SQMs for the inverted pendulum control problem

\begin{tabular}{|c|c|c|c|c|}
\hline Very small & small & $\boldsymbol{W}$ & large & Very large \\
\hline 0.125 & 0.25 & 0.5 & 0.75 & 0.875 \\
\hline
\end{tabular}

The configuration of the FCHA is shown in Fig. 7.

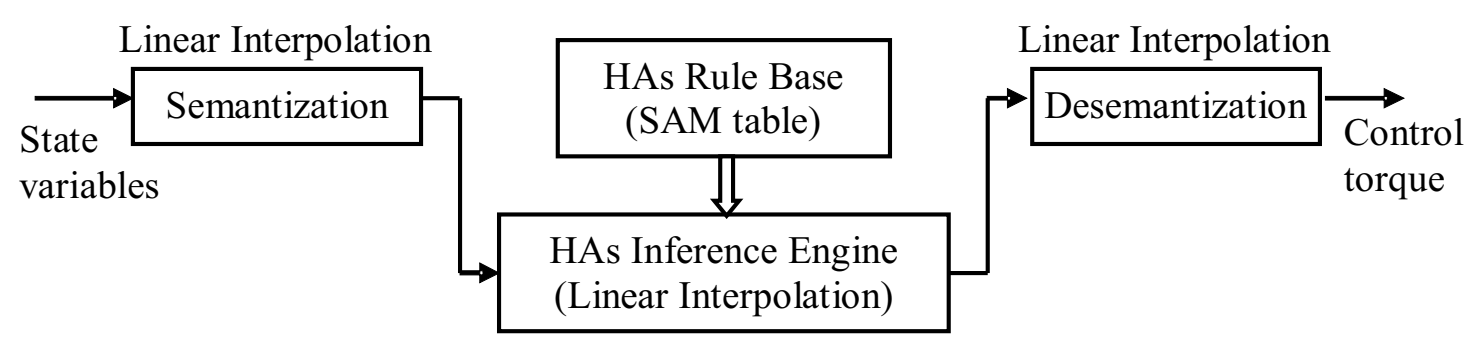

Fig. 7. The configuration of the FCHA

\subsubsection{Semantization and Desemantization}

Note that, for convenience in presenting the quantitative semantics formalism in studying the meaning of vague terms, we have assumed that the common reference domain of the linguistic variables is the interval $[0,1]$, called the semantic domain of the linguistic variables. In applications, we need use the values in the reference domains, e.g. the interval $[a, b]$, of the linguistic variables and, therefore, we have to transform the interval $[a, b]$ into $[0,1]$ and, vice-versa. The transformation of the interval $[a, b]$ into $[0,1]$ 
is called a semantization and its converse transformation from $[0,1]$ into $[a, b]$ is called a desemantization. The new terminology "semantization" was defined and accepted in Ho et al. $[15]$.

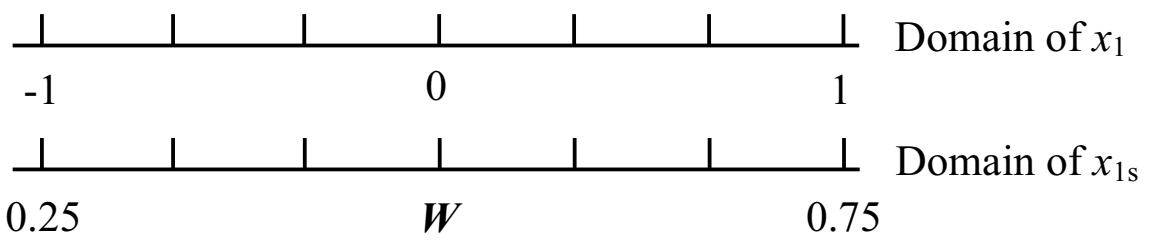

Fig. 8. Transformation: $x_{1}$ to $x_{1 s}$

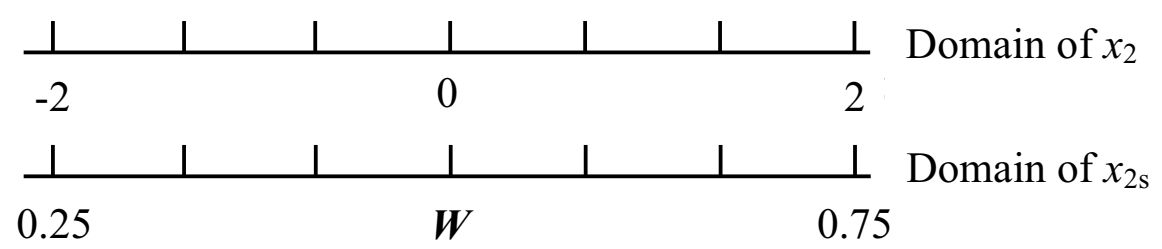

Fig. 9. Transformation: $x_{2}$ to $x_{2 s}$

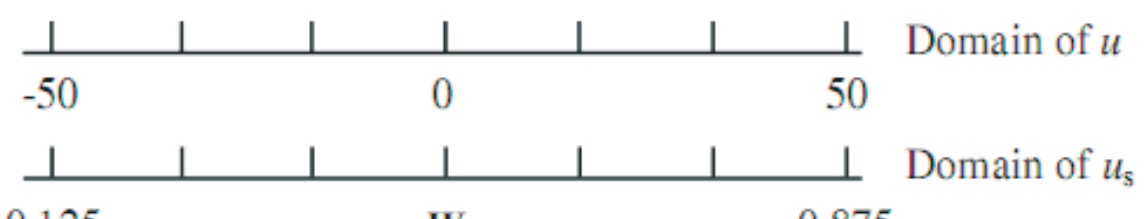

$\begin{array}{lll}0.125 & W & 0.875\end{array}$

Fig. 10. Transformation: $u$ to $u_{s}$

The semantizations for each state variable are defined by the transformations given in Fig. 8 and Fig. 9. The semantization and desemantization for the control variable are defined by the transformation given in Fig. $10\left(x_{1}, x_{2}\right.$ and $u$ are replaced with $x_{1 s}, x_{2 s}$ and $u_{s}$ when transforming from real domain to semantic one, respectively).

\subsubsection{HAs rule base}

We have the SAM (semantic associative memory) table based on FAM one (Table 1) with semantically quantifying mappings as shown in Table 4.

\subsubsection{HAs inference engine}

The Quantifying Semantic Curves describing the HAs inference method are established through the points that present the control rules occurring in Table 4 as shown in Fig. 11. Hence, $u_{s}$ is determined by linear interpolations through $x_{1 s}$ and $x_{2 s}$. 
Table 4. SAM table with semantically quantifying mappings

\begin{tabular}{|c|c|c|c|}
\hline \multirow{2}{*}{$x_{1 s}$} & \multicolumn{3}{|c|}{$x_{2 s}$} \\
\cline { 2 - 4 } & small: 0.25 & $\boldsymbol{W}: 0.5$ & large $: 0.75$ \\
\hline small: 0.25 & Very small: 0.125 & small: 0.25 & $\boldsymbol{W}: 0.5$ \\
\hline $\boldsymbol{W}: 0.5$ & small: 0.25 & $\boldsymbol{W}: 0.5$ & large $: 0.75$ \\
\hline large: 0.75 & $\boldsymbol{W}: 0.5$ & large: 0.75 & Very large: 0.875 \\
\hline
\end{tabular}

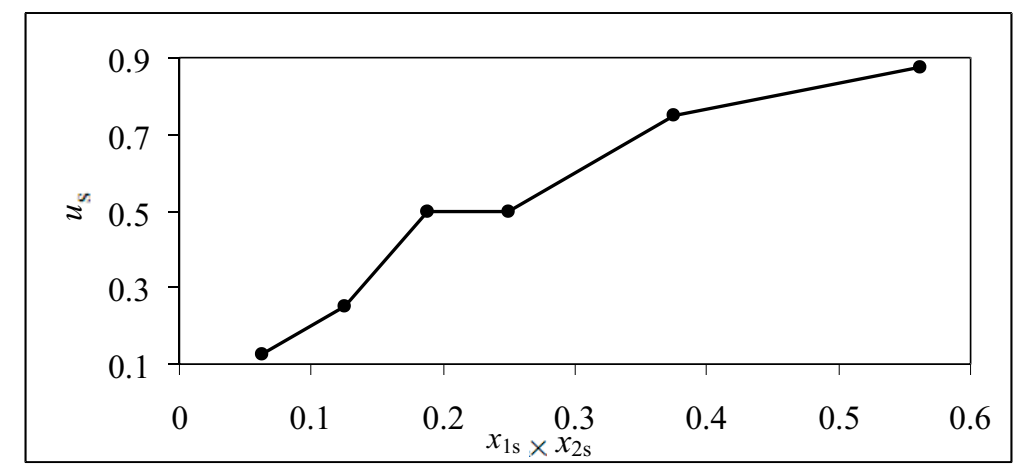

Fig. 11. Quantifying Semantic Curve

\subsection{Optimal fuzzy control using hedge algebras (OFCHA) of the inverted pendulum}

The values of linguistic variables shown in Table 3 are now considered as design variables, their intervals are determined as follow (Table 5):

Table 5. The interval parameters of SQMs

\begin{tabular}{|c|c|c|c|c|}
\hline [Very small] & [small] & {$[\boldsymbol{W}]$} & [large] & [Very large] \\
\hline $0 \div 0.1875$ & $0.1876 \div 0.375$ & $0.3751 \div 0.625$ & $0.6251 \div 0.8125$ & $0.8126 \div 1$ \\
\hline
\end{tabular}

The goal function $g$ is defined as follow:

$$
g=\sum_{i=0}^{n}\left[x_{1}^{2}(i)+x_{2}^{2}(i)\right]=\min .
$$

where, $n$ is the number of control cycles. The parameters of the optimal algorithm using GA are defined as follow: number of individuals per subpopulations: NIND $=10$; number of generations: MAXGEN $=1000$; recombination probability: GGAP $=0.8$; number of variables: $\mathrm{NVAR}=5$ and fidelity of solution: $\mathrm{PRECI}=10$.

\section{RESULTS AND DISCUSSION}

In order to study the changing rule of $x_{1}(\varphi), x_{2}(\dot{\varphi})$ and $u$ using CFC, FCHA and OFCHA, the following numerical values are used for simulations: $\gamma=0.5, m=2 \mathrm{~kg}, k$ $=2 \mathrm{Nm}, P_{l}=10 \mathrm{~N}, P_{2}=1 \mathrm{~N}, c=0.005 \mathrm{Nms}, \omega=\pi / 2, g=9.81 \mathrm{~m} / \mathrm{s}^{2}$ the sample time $\Delta t=0.1 \mathrm{~s}$. The initial conditions are $x_{1}(0)=0.6 \mathrm{rad}$ and $x_{2}(0)=1 \mathrm{rad} / \mathrm{s}$. The values of 
$x_{1}, x_{2}, u$ and $e$ obtained from OFCHA, FCHA, CFC corresponding to differential values of pendulum length are shown in following subsections. The error $e$, which measures the performance of the controllers, is defined [15]:

$$
e=\sqrt{\varphi^{2}+\dot{\varphi}^{2}}=\sqrt{x_{1}^{2}+x_{2}^{2}}
$$

\subsection{Results corresponding to $1=1.5 \mathrm{~m}$}

The optimal parameters of SQMs for OFCHA $(l=1.5 \mathrm{~m})$ is tabulated in Table 6 . The values of $x_{1}, x_{2}, u$ and $e$ obtained from OFCHA, FCHA, CFC are shown in Figs. $12-15$.

Table 6. Optimal parameters of SQMs for OFCHA, $l=1.5 \mathrm{~m}$

\begin{tabular}{|c|c|c|c|c|}
\hline Very small $_{o p}$ & small $_{o p}$ & $\boldsymbol{W}_{o p}$ & large $_{o p}$ & Very large $_{o p}$ \\
\hline 0.177786 & 0.193645 & 0.429330 & 0.767253 & 0.874883 \\
\hline
\end{tabular}

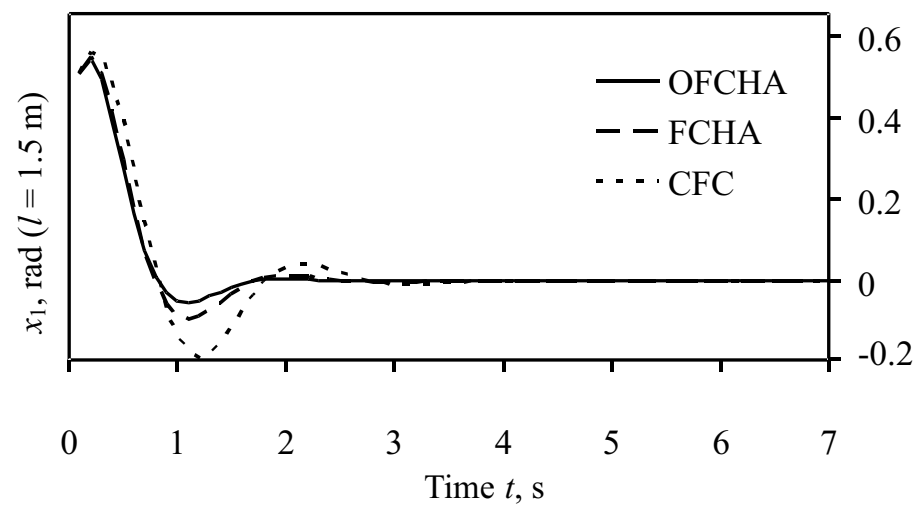

Fig. 12. The angle $x_{1}, \operatorname{rad}(l=1.5 \mathrm{~m})$

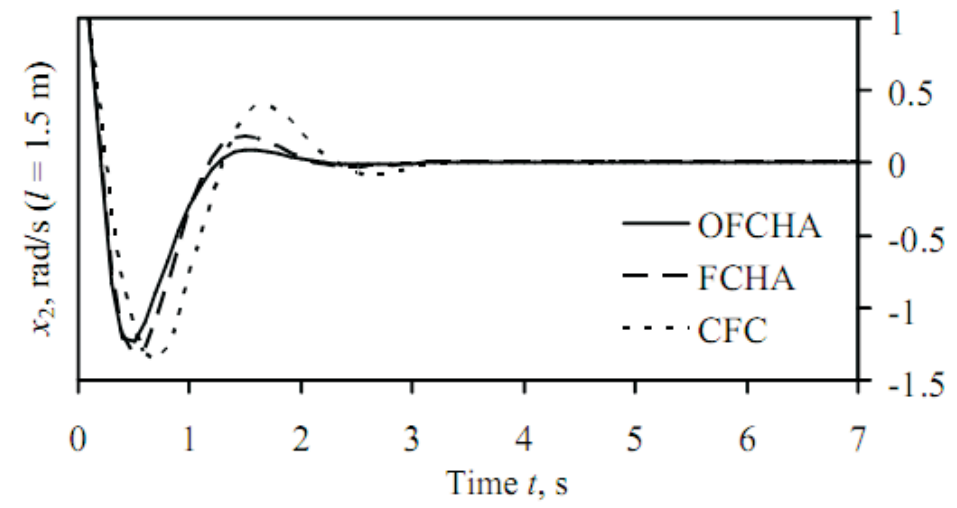

Fig. 13. The angular velocity $x_{2}, \mathrm{rad} / \mathrm{s}(l=1.5 \mathrm{~m})$ 


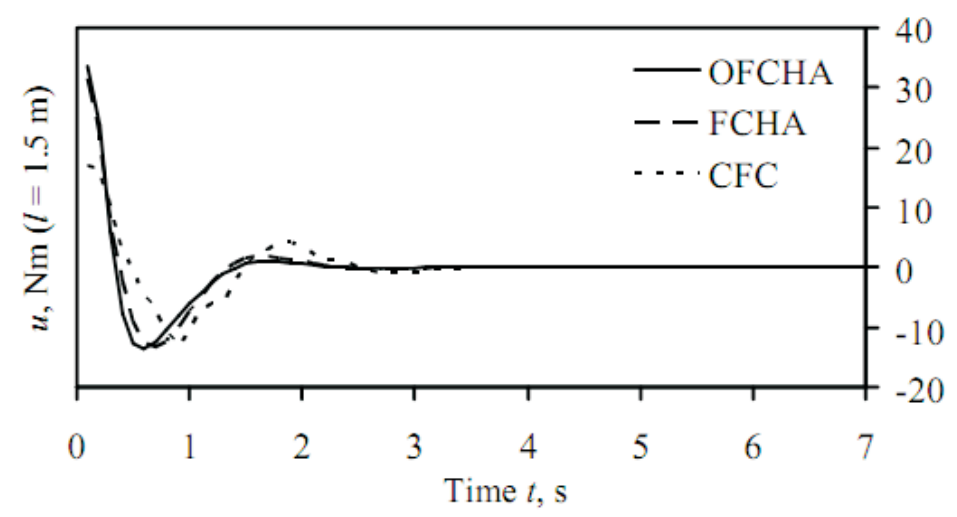

Fig. 14. The control torque $u, \mathrm{Nm}(l=1.5 \mathrm{~m})$

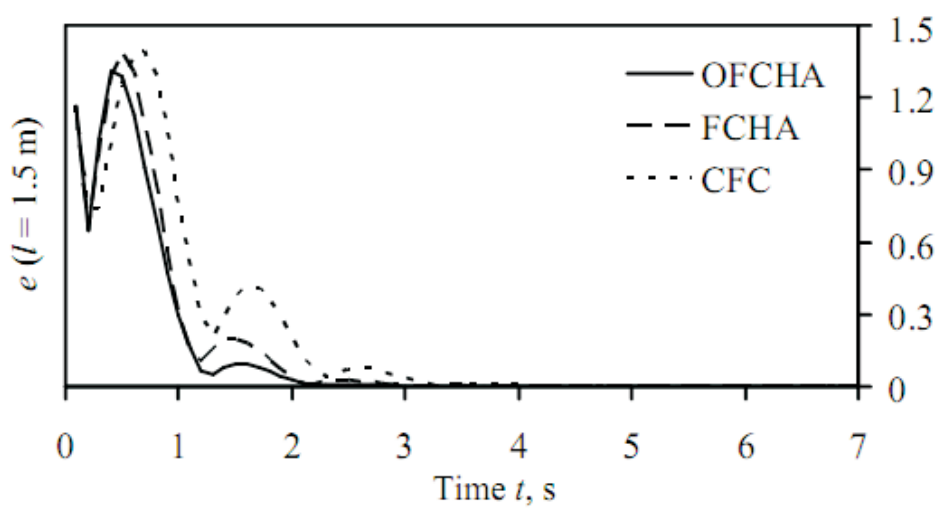

Fig. 15. The error $e(l=1.5 \mathrm{~m})$

As can be seen from Fig. 12-15, the results obtained from OFCHA are better than those of FCHA and CFC, the system is stabilized in about $3 \mathrm{~s}$ corresponding to OFCHA and FCHA and about $4 \mathrm{~s}$ corresponding to $\mathrm{CFC}$ although a small vibration appears during the stabilization process.

\subsection{Results corresponding to $1=2 \mathrm{~m}$}

The optimal parameters of SQMs for OFCHA $(l=2 \mathrm{~m})$ is tabulated in Table 7 . The values of $x_{1}, x_{2}, u$ and $e$ obtained from OFCHA, FCHA, CFC corresponding to $l=$ $2 \mathrm{~m}$ are shown in Fig. 16-19.

Table 7. Optimal parameters of SQMs for OFCHA, $l=2 \mathrm{~m}$

\begin{tabular}{|c|c|c|c|c|}
\hline Very small $_{o p}$ & small $_{o p}$ & $\boldsymbol{W}_{o p}$ & large $_{o p}$ & Very large $_{o p}$ \\
\hline 0.187500 & 0.187600 & 0.486248 & 0.799677 & 0.814249 \\
\hline
\end{tabular}




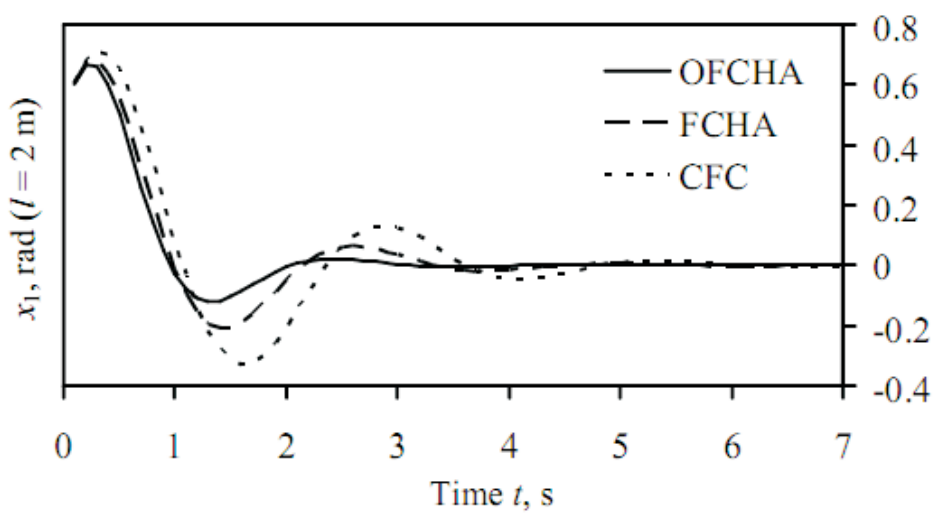

Fig. 16. The angle $x_{1}, \operatorname{rad}(l=2 \mathrm{~m})$

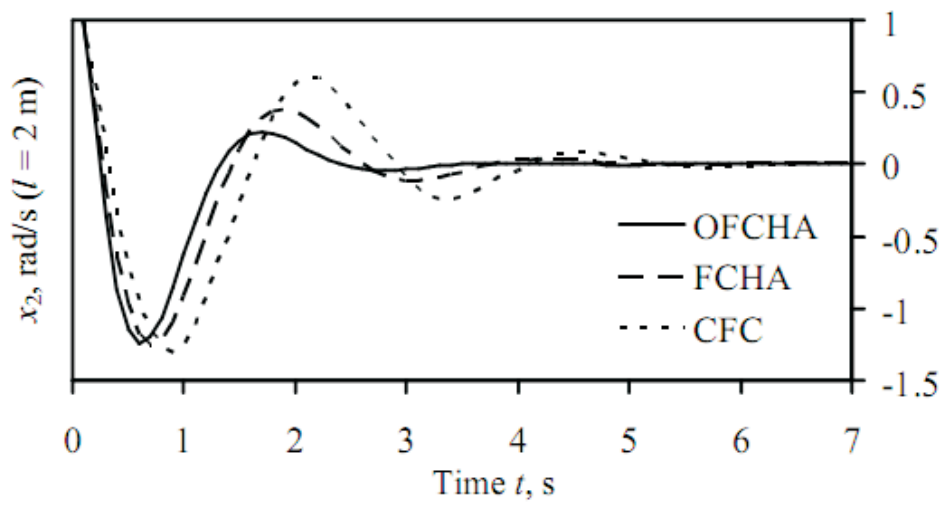

Fig. 17. The angular velocity $x_{2}, \mathrm{rad} / \mathrm{s}(l=2 \mathrm{~m})$

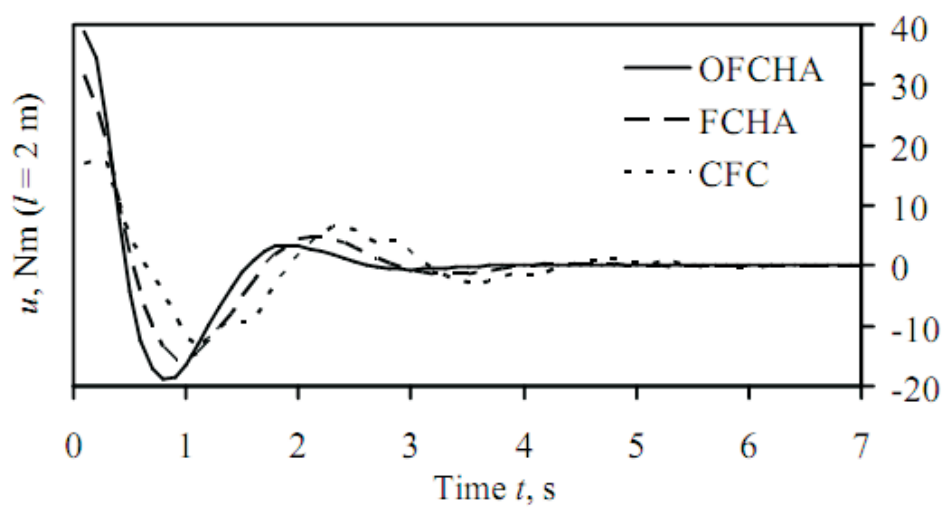

Fig. 18. The control torque $u, \mathrm{Nm}(l=2 \mathrm{~m})$ 


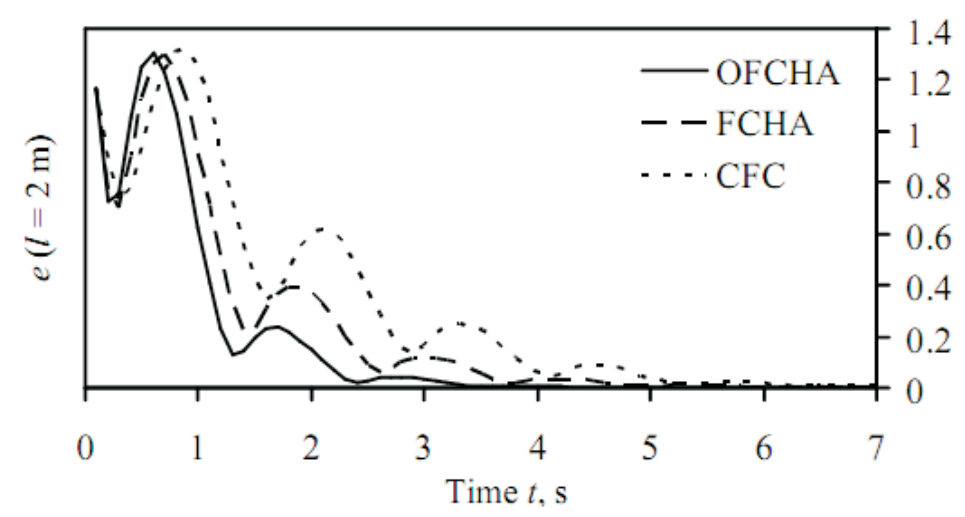

Fig. 19. The error $e(l=2 \mathrm{~m})$

From Figs. 16-19, the results obtained from OFCHA are better than those of FCHA and CFC, the system is stabilized in over $4 \mathrm{~s}$ corresponding to OFCHA, over $6 \mathrm{~s}$ corresponding to FCHA and over $7 \mathrm{~s}$ corresponding to $\mathrm{CFC}$ although a small vibration appears during the stabilization process.

\subsection{Discussion}

From Tables 2 and 3, it can be conceded that the semantic order of HAFC is always guaranteed.

The semantization method of FCHA (Figs. 8-10) is simpler than the fuzzification method of CFC (Figs. 4-6).

The desemantization method of FCHA, executed by linear interpolations (see Fig. 10), is simpler than the centroid defuzzification method of CFC very much.

The inference method of FCHA, executed by linear interpolations (see Fig. 11), is simpler than the one of CFC (Mamdani method - in this paper) very much.

FCHA, a new fuzzy control algorithm, does not require fuzzy sets to provide the semantics of the linguistic terms used in the fuzzy rule system rather the semantics is obtained through the semantically quantifying mappings (SQMs). In the algebraic approach, the design of an FCHA leads to the determination of the parameter of SQMs, which are the fuzziness measure of primary terms and linguistic hedges occurring in the fuzzy model.

\section{CONCLUSIONS}

In the present work, a new fuzzy controller based on HAs is applied for the stabilization control of a damped elastic jointed inverted pendulum with periodic follower force. The main results are summarized as follows:

The algebraic approach to term-domains of linguistic variables is quite different from the fuzzy sets one in the representation of the meaning of linguistic terms and the methodology of solving the fuzzy multiple conditional reasoning problems.

It is obtained that HAFC is simpler, effective and more understandable in comparison with CFC for controlling the above pendulum. 
In fuzzy logic, many important concepts like fuzzy set, T-norm, S-norm, intersection, union, complement, composition... are used in approximate reasoning. This is an advantage for the process of flexible reasoning, but there are too many factors such as shape and number of membership functions, defuzzification method,... influencing the precision of the reasoning process and it is difficult to optimize. Those are subjective factors that cause error in determining the values of control process. Meanwhile, approximate reasoning based on hedge algebras, from the beginning, does not use fuzzy set concept and its precision is obviously not influenced by this concept. Therefore, the method based on hedge algebras does not need to determine shape and number of membership function, neither does it need to solve defuzzification problem. Besides, in calculation, while there is a large number of membership functions, the volume of calculation based on fuzzy control increases quickly, meanwhile the volume of calculation based on hedge algebras does not increase much with very simple calculation. With these above advantages, it is definitely possible to use hedge algebras theory for many different controlling problems.

This is our first research in the field of structural active control using FCHA, needed problems when establishing a new controller such as the stability and robustness of the algorithm will be mentioned in our further researches.

\section{REFERENCES}

[1] Yi, J. and Yubazaki, N., Stabilization fuzzy control of inverted pendulum systems, Artificial Intelligence in Engineering, 14, (2000), 153-163.

[2] Yi, J., Yubazaki, N. and Hirota, K., A new fuzzy controller for stabilization of parallel-type double inverted pendulum system, Fuzzy Sets and Systems, 126, (2002), 105-119.

[3] Becerikli, Y. and Celikb, B. K., Fuzzy control of inverted pendulum and concept of stability using Java application, Mathematical and Computer Modelling, 46, (2007), 24-37.

[4] Tao, C. W., Taur, J. S., Wanga, C. M. and Chena, U. S., Fuzzy hierarchical swing-up and sliding position controller for the inverted pendulum-cart system, Fuzzy Sets and Systems, 159, (2008), 2763-2784.

[5] Li, Z. and Xu, C., Adaptive fuzzy logic control of dynamic balance and motion for wheeled inverted pendulums, Fuzzy Sets and Systems, 160, (2009), 1787-1803.

[6] Sinha, S. C. and Butcher, E. A., Symbolic computation of fundamental solution matrices for linear time-periodic dynamical systems, Journal of Sound and Vibration, 206(1), (1997), 61-85.

[7] Galán, J., Fraser, W. B., Acheson, D. J. and Champneys, A. R., The parametrically excited upside-down rod: an elastic jointed pendulum model, Journal of Sound and Vibration, 280, (2005), 359-377.

[8] Ma, H. and Butcher, E. A., Stability of elastic columns with periodic retarded follower forces, Journal of Sound and Vibration, 286, (2005), 849-867.

[9] Mailybaev, A. A. and Seyranian, A. P., Stabilization of statically unstable systems by parametric excitation, Journal of Sound and Vibration, 323, (2009), 1016-1031.

[10] Ho, N. C. and Wechler, W., Hedge algebras: an algebraic approach to structure of sets of linguistic truth values, Fuzzy Sets and Systems, 35, (1990), 281-293.

[11] Ho, N. C. and Wechler, W., Extended hedge algebras and their application to fuzzy logic, Fuzzy Sets and Systems, 52, (1992), 259-281.

[12] Ho, N. C. and Nam, H. V., An algebraic approach to linguistic hedges in Zadeh's fuzzy logic, Fuzzy Sets and Systems, 129, (2002), 229-254. 
[13] Ho, N. C., A topological completion of refined hedge algebras and a model of fuzziness of linguistic terms and hedges, Fuzzy Sets and Systems, 158, (2007), 436-451.

[14] Ho, N. C. and Long, N. V., Fuzziness measure on complete hedge algebras and quantifying semantics of terms in linear hedge algebras, Fuzzy Sets and Systems, 158, (2007), 452-471.

[15] Ho, N. C., Lan, V. N. and Viet, L. X., Optimal hedge-algebras-based controller: Design and application, Fuzzy Sets and Systems, 159, (2008), 968-989.

[16] Chapra, S. C. and Canale, R. P., Numerical Methods for Engineers, McGraw-Hill, New York, USA, (2006).

[17] Ross, T. J., Fuzzy logic with engineering applications, John Wiley \& Sons, England, (2004).

Received February 1, 2010

\section{ĐIỀU KHIỂN MỜ TỐI ƯU SỬ DỤNG ĐẠI SỐ GIA TỬ CHO HỆ CON LẮC NGƯợC LIÊN KẾT ĐÀN HỒI - GIẢM CHẤN}

Trong bài báo này, ba bộ điều khiển gồm OFCHA (điều khiển mờ tối ưu sử dụng đại số gia tử), FCHA (điều khiển mờ sử dụng đại số gia tử) và $\mathrm{CFC}$ (điều khiển mờ thông thường) được thiết kế để đưa một con lắc ngược có liên kết đàn hồi - giảm chấn chịu tải theo tuần hoàn theo thời gian về vị trí cân bằng theo phương thẳng đứng. Những giá trị khác nhau của chiều dài con lắc được khảo sát trong nghiên cứu. Các kết quả mô phỏng được đưa ra nhằm so sánh hiệu quả của $\mathrm{OFCHA}$ so với $\mathrm{FCHA}$ và $\mathrm{CFC}$. 